POS PROCEEDINGS

\title{
Search for heavy bosons and lepton flavour violation with dileptons at CMS
}

\author{
Hwi Dong Yoo* \\ on behalf of the CMS Collaboration \\ Seoul National University, Republic of Korea \\ E-mail: hdyoo@cern.ch
}

\begin{abstract}
We report the results for various heavy boson searches in the dilepton channel at CMS. All results are based on proton-proton collision data at $\sqrt{s}=13 \mathrm{TeV}$ collected by the CMS detector in 2015 and 2016. No excess of events over the standard model prediction is observed and upper limits for various benchmark models are calculated.
\end{abstract}

38th International Conference on High Energy Physics 3-10 August 2016

Chicago, USA

${ }^{*}$ Speaker. 


\section{Introduction}

Many beyond the Standard Model (BSM) models predict the existence of new heavy particles which can decay into leptons. These leptonic channels provide cleaner signatures compared to hadronic decays, however the branching fractions are relatively smaller. The most popular channels in the leptonic decays are the electron (e) and muon $(\mu)$ channels (and their combination) as their experimental uncertainties are smaller than others. The $\tau$ channel is treated separately because the $\tau$ reconstruction is different from $e$ and $\mu$. $v$ 's leave a signature as missing transverse energy (MET) in the CMS detector [1].

Searches presented in this paper probe a large range of energy up to very high mass region, from few hundred $\mathrm{GeV}$ to few $\mathrm{TeV}$. Due to such large coverage of the mass region, searches are usually very sensitive to the center-of-mass energy. Discovery potential of these heavy particles is increased dramatically at $\sqrt{s}=13 \mathrm{TeV}$ compared to $\sqrt{s}=8 \mathrm{TeV}$ and the results based on $13 \mathrm{TeV}$ dataset collected in 2015 corresponding to an integrated luminosity of $2.8 \mathrm{fb}^{-1}$ make the results with $8 \mathrm{TeV}$ dataset obsolete.

In this paper, we present new results at $13 \mathrm{TeV}$ with 2015 and 2016 datasets. The integrated luminosity used in the results corresponds to around $3 \mathrm{fb}^{-1}$ and $13 \mathrm{fb}^{-1}$ for 2015 and 2016 datasets respectively.

\section{Search for $\mathbf{Z}^{\prime}$ and $\mathbf{W}^{\prime}$}

Searches for a $Z^{\prime}$ resonance are performed using a model independent shape-based analysis with a narrow resonance. Where further interpretations of high mass dilepton is performed [2]. Standard CMS high $\mathrm{p}_{\mathrm{T}}$ muon and electron identification and event selection are used to select dilepton $Z^{\prime}$ candidates. The dominant backgrounds are from Drell-Yan (DY), tit, and diboson (WW, WZ and ZZ) events. In addition, jets misidentified as leptons (W+jets and dijet) in the dielectron channel are estimated. Figure 1 shows the comparison plots of dielectron (left) and dimuon (right) invariant mass spectra between data and Standard Model (SM) background predictions which are mostly based on MC samples.
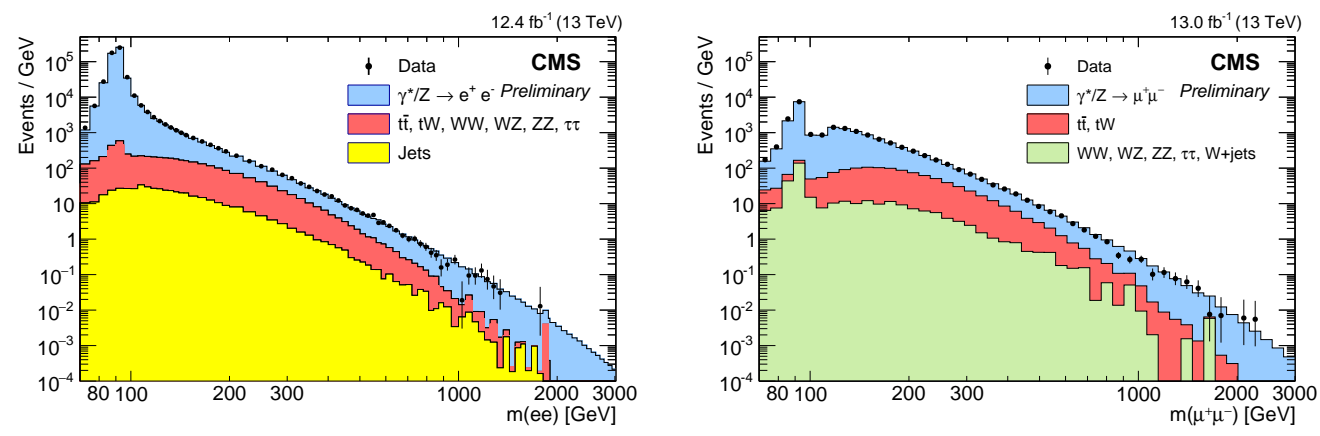

Figure 1: Dielectron (left) and dimuon (right) invariant mass spectra after event selection.

No excess beyond the SM background predictions is observed. The limits are calculated using the ratio between high mass spectrum normalized by the yield at $\mathrm{Z}$ peak. The statistical interpre- 
tation is computed using the spin $-1 \mathrm{Z}^{\prime}$ resonance with two theoretical models, $\mathrm{Z}_{\psi}^{\prime}$ (width is $0.6 \%$ ) and $Z_{S S M}^{\prime}$ (width is 3\%) at $95 \%$ confidence level (CL). Figure 2 shows the results of limit calculation with the $Z^{\prime}$ models in dielectron (left), dimuon (middle) and combined (right) channels. $Z_{\psi}^{\prime}$ and $Z_{S S M}^{\prime}$ models with masses less than $3.5 \mathrm{TeV}$ and $4.0 \mathrm{TeV}$ respectively are excluded in the combination of dielectron and dimuon channels. A Search for $Z^{\prime}$ decaying into $\tau$ lepton pairs is also studied in Ref. [4].
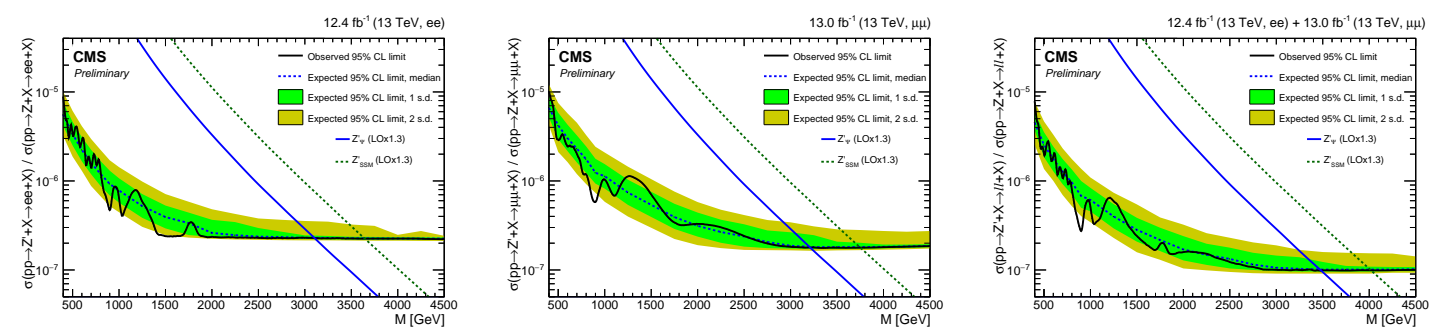

Figure 2: The 95\% CL upper limits on for $Z^{\prime}$ resonance models using the ratio of production cross section between $Z^{\prime}$ resonance and $\mathrm{Z}$ boson masses for the dielectron (left), dimuon (middle), and combined (right) channels.

A similar search for a high mass resonance in lepton flavor violation channel is performed [3]. Several extensions of the SM predict such high mass resonance decaying into the $e \mu$ final state, such as R-parity violation (RPV) with $\tilde{v}_{\tau}$ and quantum black holes (QBH). We select $e \mu$ pair in the final state with similar electron and muon identification criteria used in the search for $Z^{\prime}$ in dielectron and dimuon channels described above. The dominant SM backgrounds are from t⿱t, $\mathrm{DY}$ and diboson events decaying into two prompt leptons. Other backgrounds based on the nonprompt lepton or misidentified from jets also have a nonnegligible contribution and they are estimated based on control samples in data. No significant excess over the SM prediction is observed in the $e \mu$ invariant mass spectrum after all event selection. Limits on the signal cross section times branching fraction are set for the RPV and QBH benchmark models. Figure 3 shows the invariant $e \mu$ mass spectrum for data compared to SM background expectation on the left and 95\% CL upper limit for RPV $\tilde{v}_{\tau}$ on the right.

Single-lepton based search are also performed in addition to dilepton-based searches using $\sqrt{s}=13 \mathrm{TeV}$ dataset. A search for new physics such as the $\mathrm{W}^{\prime}$ in which events contain a lepton $(\mathrm{e}, \mu, \tau)$ and MET introduced by $v$ not interacting with the CMS detector is studied [5, 6]. A new heavy gauge boson, $\mathrm{W}^{\prime}$, can appear in many BSM models such as Sequential SM (SSM), RandalSundrum gravitons, composite Higgs etc. We require one isolated high $\mathrm{p}_{\mathrm{T}}$ lepton with large MET in an event. To suppress SM backgrounds from other dilepton processes like DY, we veto on additional leptons. The dominant SM background comes from $\mathrm{W}$ boson decaying into a lepton and MET. The main discriminant variable used in this search is the transverse invariant mass, $\mathrm{M}_{\mathrm{T}}$, defined as $\mathrm{M}_{\mathrm{T}}=\sqrt{2 \mathrm{p}_{\mathrm{T}}^{\ell} \mathrm{E}_{\mathrm{T}}^{\text {miss }}\left(1-\cos \left[\Delta\left(\overrightarrow{\mathrm{p}}_{\mathrm{T}}^{\ell}, \overrightarrow{\mathrm{p}}_{\mathrm{T}}^{\text {miss }}\right)\right]\right.}$, where $\mathrm{p}_{\mathrm{T}}^{\ell}$ is lepton $\mathrm{p}_{\mathrm{T}}, \mathrm{E}_{\mathrm{T}}^{\text {miss }}$ is MET of $v, \overrightarrow{\mathrm{p}}_{\mathrm{T}}^{\text {miss }}$ is $-\Sigma \overrightarrow{\mathrm{p}}_{\mathrm{T}}$ of all reconstructed particles with MET. Using this discriminant variable, we look for signal events beyond the SM background prediction at very high $\mathrm{M}_{\mathrm{T}}$ values.

No significant deviation from SM background predictions is observed in the $\mathrm{M}_{\mathrm{T}}$ and therefore limits are calculated in the context of the SSM $\mathrm{W}^{\prime}$ for both e or $\mu+\mathrm{MET}$ and $\tau+\mathrm{MET}$ channels 

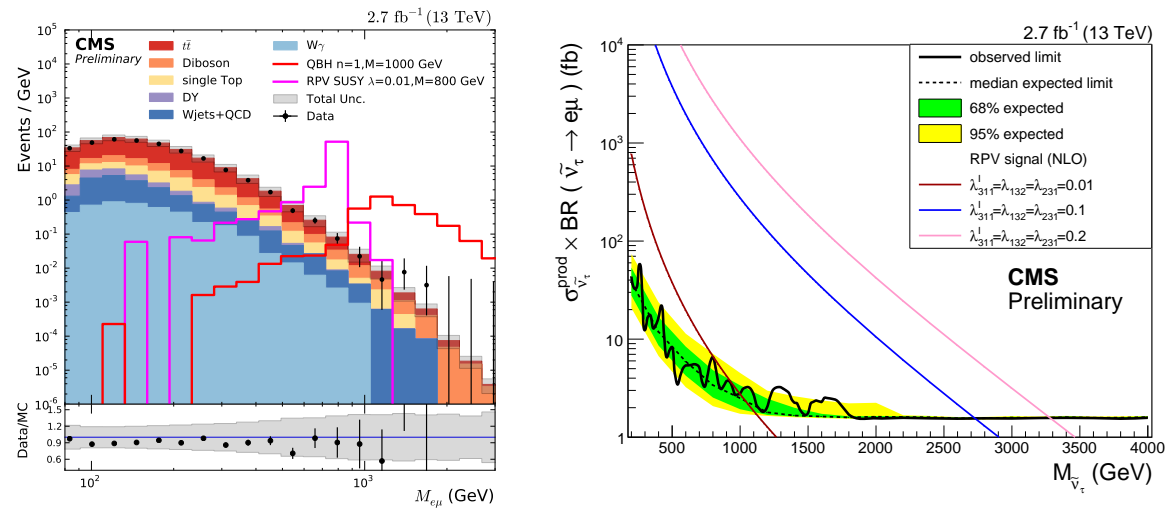

Figure 3: Left: The invariant mass spectrum of $e \mu$ pair candidates after event selection. Right: $95 \% \mathrm{CL}$ upper limit on the signal cross section times branching fraction for RPV $\tilde{v}_{\tau}$.

respectively. Figure 4 shows the results for the limits on the signal cross section times branching fraction for the combination of e+MET and $\mu+$ MET channels (left) and the $\tau+\mathrm{MET}$ (right) channel.
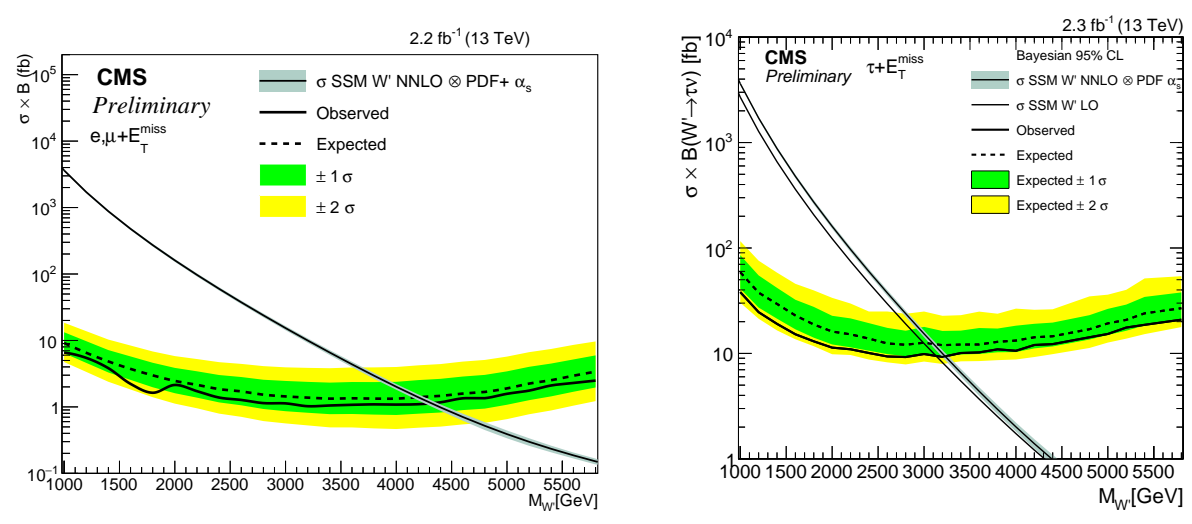

Figure 4: Observed 95\% CL upper limits for the combination of the electron and muon channels (left) and the tau channel (right).

\section{Search for $\mathrm{Z} \gamma$ resonances}

As a second part, we present a search for high-mass resonances decaying into $\mathrm{Z} \gamma$ final states [7, 8]. In the search, a $\mathrm{Z}$ boson can decay into a dilepton or dijet. The dilepton channel provides a relatively cleaner event signature than the dijet channel, however the branching fraction is larger in dijet channel. Moreover the dilepton channel can cover relatively lower mass regions (due to trigger allowance), and the dijet channel covers relatively higher mass regions (due to higher acceptance). Therefore two channels have compliment each other in searching for a high-mass scalar X decaying into $\mathrm{Z} \gamma$. In the dilepton channel, we only consider $\mathrm{Z}$ decaying into $e^{+} e^{-}$or $\mu^{+} \mu^{-}$.

This search in the dilepton channel is performed from $\mathrm{M}(\mathrm{Z} \gamma)>300 \mathrm{GeV}$ up to $2000 \mathrm{GeV}$. To estimate backgrounds, we fit the data with background modeling, similar to the technique used in 
diphoton searches [9]. Events for dielectron or dimuon associated with one photon are selected, after applying standard CMS identification for electrons, muons and photons. Also dielectron or dimuon selection should be placed in $\mathrm{Z}$ resonance mass window to suppress other backgrounds. Figure 5 shows the $e^{+} e^{-} \gamma$ (left) and $\mu^{+} \mu^{-} \gamma$ (right) invariant mass spectra for data after event selection. The red colour shows the fit results on the background from the data distribution.
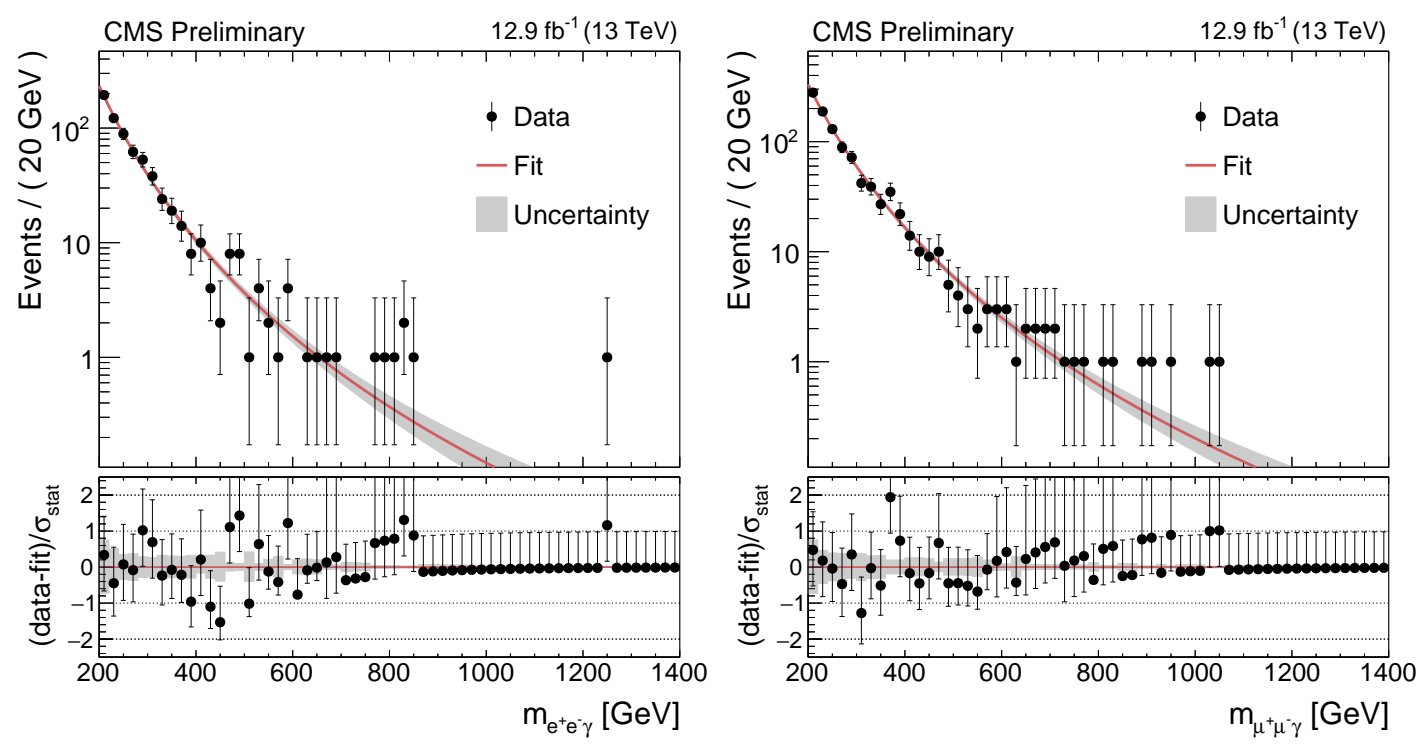

Figure 5: Observed the $e^{+} e^{-} \gamma$ (left) and $\mu^{+} \mu^{-} \gamma$ (right) invariant mass spectra at the $13 \mathrm{TeV}$ data.

No significant excess is observed beyond the SM background expectation. We calculate the upper limits on the signal production cross section times branching fraction for $\mathrm{X} \rightarrow \mathrm{Z} \gamma$. The results of dielectron and dimuon channels are combined and the result is shown in the Figure 6 (left).

In the dijet channel, a similar bump search in $M(Z \gamma)$ spectrum is also performed. One remarkable thing is that the boosted topology ( $\mathrm{Z} \rightarrow \mathrm{J}$, where $\mathrm{J}$ is a jet substructure) has been used to improve the ratio of signal over background. A b-tagging is exploited to discriminate backgrounds further in the final state. Similar quality of event selection is applied to select good quality dijet events associated with a photon. Backgrounds are fit on the $\mathrm{M}(\mathrm{Z} \gamma)$ distribution from data after event selection and no visible excess beyond the SM backgrounds is observed. Figure 6 (right) plot shows the limit calculation results as a function of $\mathrm{M}(\mathrm{Z} \gamma)$ for various dataset.

\section{Summary}

In summary, we have discussed the latest CMS results of the search for heavy bosons in dilepton channels at $13 \mathrm{TeV}$ in proton-proton collisions delivered by LHC in 2015 and 2016. No significant deviation from the SM predictions is observed and therefore limits are set at 95\% CL with various benchmark models. 

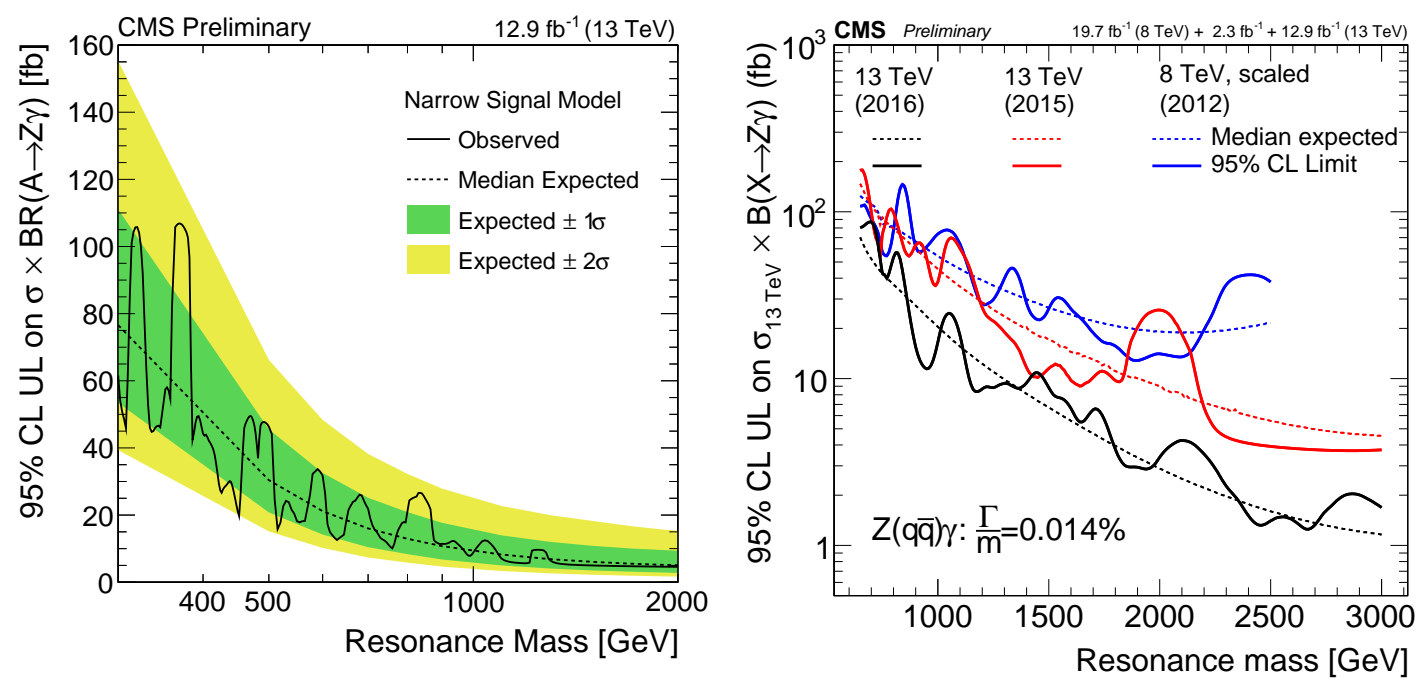

Figure 6: $95 \% \mathrm{CL}$ upper limit on the signal cross section times branching fraction for $\mathrm{X} \rightarrow \mathrm{Z} \gamma$ obtained at $13 \mathrm{TeV}$. Left (right) plot shows the results for $\mathrm{Z} \rightarrow \ell \ell(\mathrm{jj})$ channel.

\section{Acknowledgments}

H. D. Yoo is supported in part by the National Research Foundation of Korea (NRF) funded by the Korea government (NRF-2015R1C1A1A01053087 and NRF-2015R1A4A1042542).

\section{References}

[1] CMS Collaboration, "The CMS experiment at the CERN LHC”, JINST 3 (2008) S08004.

[2] CMS Collaboration, "Search for a high-mass resonance decaying into a dilepton finale state in 13 $\mathrm{fb}^{-1}$ of pp collisions at $\sqrt{s}=13 \mathrm{TeV}$ ", CMS-PAS-EXO-16-031, cds.cern.ch/record/2205764.

[3] CMS Collaboration, "Search for high-mass resonances and quantum black holes in the e $\mu$ final state in proton-proton collisions at $\sqrt{s}=13 \mathrm{TeV}$ ", CMS-PAS-EXO-16-001, cds.cern.ch/record/2149046.

[4] CMS Collaboration, "Search for new physics with high-mass $\tau$ lepton pairs in pp collisions at $\sqrt{s}=13 \mathrm{TeV}$ with the CMS detector", CMS-PAS-EXO-16-008, cds.cern.ch/record/2160363.

[5] CMS Collaboration, "Search for SSM W' production, in the lepton+MET final state at a center-of-mass energy of 13 TeV”, CMS-PAS-EXO-15-006, cds.cern.ch/record/2114864.

[6] CMS Collaboration, "Search for $\mathrm{W}^{\prime}$ decaying to tau lepton and neutrino in proton-proton collisions at $\sqrt{s}=13$ TeV”, CMS-PAS-EXO-16-006, cds.cern.ch/record/2140976.

[7] CMS Collaboration, "Search for high-mass resonances in $\mathrm{Z} \gamma \rightarrow \mathrm{e}^{+} \mathrm{e}^{-} \gamma / \mu^{+} \mu^{-} \gamma$ final states in proton-proton collisions at $\sqrt{s}=13 \mathrm{TeV}$ with $12.9 \mathrm{fb}^{-1}$,, CMS-PAS-EXO-16-034, cds.cern.ch/record/2204921.

[8] CMS Collaboration, "Search for high-mass resonances in $\mathrm{Z}(\mathrm{q} \overline{\mathrm{q}}) \gamma$ final state in pp collisions at $\sqrt{s}=13 \mathrm{TeV}$ with $12.9 \mathrm{fb}^{-1}$, CMS-PAS-EXO-16-035, cds.cern.ch/record/2205280.

[9] CMS Collaboration, "Search for Resonant Production of High-Mass Photon Pairs in proton-proton collisions at $\sqrt{s}=8$ and 13 TeV” Phys. Rev. Lett. 117 (2016) 051802. 